\title{
Criminologie
}

\section{Classes dominantes, classes délinquantes ?}

Le crime économique comme figure de la critique sociale

Ruling classes, criminal classes?

White-collar crime as figure of social critique

\section{Clases dominantes, clases delincuentes?}

\section{El crimen económico como una figura de la crítica social}

\section{Jean Bérard}

Volume 49, numéro 1, printemps 2016

Quand le crime économique contribue au développement des sciences sociales

URI : https://id.erudit.org/iderudit/1036200ar

DOI : https://doi.org/10.7202/1036200ar

Aller au sommaire du numéro

Éditeur(s)

Les Presses de l’Université de Montréal

ISSN

0316-0041 (imprimé)

1492-1367 (numérique)

Découvrir la revue

Citer cet article

Bérard, J. (2016). Classes dominantes, classes délinquantes ? Le crime économique comme figure de la critique sociale. Criminologie, 49(1), 203-224. https://doi.org/10.7202/1036200ar

\section{Résumé de l'article}

L’article aborde la question de la délinquance des élites en déplaçant l'attention des travaux criminologiques vers les travaux des économistes et sociologues critiques, principalement français, qui portent sur les comportements des classes dominantes. La problématique porte sur les analyses formulées pour rendre compte de la crise actuelle en incriminant le comportement des classes dominantes. L'article étudie la mobilisation du vocabulaire de la déviance pour qualifier des comportements économiques et politiques de vol, d'extorsion et de violence. Il montre que ces dénonciations s'appuient sur des analyses de ces comportements comme un trait majeur des pratiques des classes dominantes contemporaines. En particulier, le renouveau de la sociologie critique s'appuie sur l'analyse du rapport singulier aux lois et aux normes des classes dominantes, en montrant qu'un de leurs traits distinctifs est de considérer ces règles comme fondamentales pour les autres, mais contournables par elles-mêmes. De telles analyses conduisent à des propositions politiques qui font usage de l'idée de sanction pénale. Mais l'article montre que ces usages sont pris dans des enjeux politiques plus larges et, en particulier, replacés dans le jeu des rapports de force entre les Etats, et entre les États et les détenteurs de capitaux qui échappent à leur contrôle.
Ce document est protégé par la loi sur le droit d'auteur. L'utilisation des services d’Érudit (y compris la reproduction) est assujettie à sa politique d'utilisation que vous pouvez consulter en ligne.

https://apropos.erudit.org/fr/usagers/politique-dutilisation/ 


\title{
Classes dominantes, classes délinquantes?
}

\section{Le crime économique comme figure de la critique sociale}

\author{
Jean Bérard \\ Professeur adjoint ${ }^{1}$ \\ École de criminologie, Université de Montréal \\ Chercheur au Centre international de criminologie comparée \\ jean.berard@umontreal.ca
}

RÉSUMÉ - L'article aborde la question de la délinquance des élites en déplaçant l'attention des travaux criminologiques vers les travaux des économistes et sociologues critiques, principalement français, qui portent sur les comportements des classes dominantes. La problématique porte sur les analyses formulées pour rendre compte de la crise actuelle en incriminant le comportement des classes dominantes. L'article étudie la mobilisation du vocabulaire de la déviance pour qualifier des comportements économiques et politiques de vol, d'extorsion et de violence. Il montre que ces dénonciations s'appuient sur des analyses de ces comportements comme un trait majeur des pratiques des classes dominantes contemporaines. En particulier, le renouveau de la sociologie critique s'appuie sur l'analyse du rapport singulier aux lois et aux normes des classes dominantes, en montrant qu'un de leurs traits distinctifs est de considérer ces règles comme fondamentales pour les autres, mais contournables par elles-mêmes. De telles analyses conduisent à des propositions politiques qui font usage de l'idée de sanction pénale. Mais l'article montre que ces usages sont pris dans des enjeux politiques plus larges et, en particulier, replacés dans le jeu des rapports de force entre les États, et entre les États et les détenteurs de capitaux qui échappent à leur contrôle.

MotS CLÉs - Criminalité économique, justice pénale, critique sociale, classes sociales, capitalisme.

1. École de criminologie et Centre international de criminologie comparée, Université de Montréal, C. P. 6128, succ. Centre-ville, Montréal (Québec), Canada, H3C 3J7. 
La Banque envisagée ainsi devient toute une politique, elle exige une tête puissante et porte alors un homme bien trempé à se mettre audessus des lois de la probité, dans lesquelles il se trouve à l'étroit.

Honoré de Balzac, La maison Nucingen, 1837

La capacité des maîtres à se maintenir dans l'esprit des lois est quasiment sans défaut, ne serait-ce que parce qu'ils les font.

Luc Boltanski, «Une étude en noir», Tracés, n 20, 2011, p. 54

La crise économique et financière a suscité une reprise d'intérêt pour l'analyse des pratiques économiques des classes dominantes, qu'elles concernent l'accumulation du capital (Piketty, 2013), le fonctionnement de la finance et des banques (Giraud, 2012; Lordon, 2008), l'évasion fiscale (Zucman, 2013) ou, plus largement, «la violence des riches» (Pinçon et Pinçon-Charlot, 2013). L'audience que ces travaux ont trouvée, sur le plan international (Piketty, 2013) ou national (on peut penser en France aux derniers ouvrages des Pinçon-Charlot), et leurs liens avec des mouvements sociaux émergents soulignent que ces analyses critiques sont en prise, d'une manière qu'il est sans doute encore difficile de déterminer, avec des transformations sociales plus profondes. Le point de départ de mon questionnement est la mobilisation par ces analyses du champ sémantique de la déviance: à la crise économique répondent des mises en accusation du fonctionnement du capitalisme sous les traits de la criminalité. La sociologie de la délinquance en col blanc a-t-elle quelque chose à apprendre de la mobilisation critique des catégories de la délinquance par des économistes et des sociologues qui œuvrent dans un autre champ? Quels sont ces usages? Font-ils signe vers des analyses classiquement associées à ces termes, en particulier l'analyse de la déviance et celle de la réaction pénale à ces déviances? $\mathrm{Ou}$ intègrent-ils ce champ sémantique au sein d'ensembles théoriques qui promeuvent d'autres outils d'analyse et, en conséquence, d'autres conceptions des réponses politiques qui peuvent être apportées?

Dans un article récent, Tombs (2015) regrette que la criminologie britannique ne se soit pas attachée de manière prioritaire à l'analyse des relations entre la grande crise financière et les pratiques frauduleuses des classes dominantes. Il note que des contributions plus importantes sont venues de l'économie et des sciences politiques et que ces disciplines ont essayé de penser des solutions qui s'écartent des formes de régulation défendues avant la crise comme suffisantes. Cet article vise à réfléchir dans cette direction en mobilisant un corpus d'auteurs, qu'on 
peut classer, pour l'essentiel, au sein de la sociologie critique et de l'économie hétérodoxe françaises. Il utilise volontairement différentes sources et registres d'écriture, des recherches universitaires aux interventions publiques, sous la forme de tribunes et entretiens, pour essayer de comprendre l'articulation entre des analyses empiriques et des positionnements politiques. L'article ne prétend pas s'appuyer sur l'ensemble des contributions disponibles ni même sur un échantillon constitué selon des critères parfaitement explicites. Je ne prétends pas non plus, loin de là, que ces analyses représentent l'ensemble des critiques politiques formulées dans les dernières années contre le fonctionnement actuel du capitalisme. Mon but est moins de faire une recension exhaustive que d'essayer de poser quelques jalons théoriques dont les liens logiques vont au-delà des auteurs mobilisés.

Cette tentative se place dans le contexte changeant et incertain de la crise du "capitalisme de déréglementation à dominante financière » (Lordon, 2008, p. 187), débutée en 2008. Ce moment de transformation est solidaire d'interrogations sur la définition sociologique des groupes sociaux placés dans les fonctions de direction. Comme le suggère Alexis Spire (2013a), dans le sillage de Bourdieu, "plutôt que de rester dépendant des ambiguités du terme de délinquance en col blanc, on propose d'utiliser plutôt celui de classe dominante, définie comme l'ensemble des agents disposant d'un pouvoir sur le fonctionnement d'un champ (politique, économique, bureaucratique ou culturel) et sur les conditions de sa reproduction» (p. 9). L'acuité de la crise financière ne doit pas conduire à opposer radicalement la période ouverte en 2007-2008 à une ère de stabilité précédente. Au contraire, pourrait-on dire, le contexte actuel conduit à la formulation d'analyses de l'histoire des transformations du capitalisme et des États (et institutions supraétatiques comme l'Union européenne) pour opérer, souvent à partir des années 1970, la généalogie à moyen terme de la situation actuelle - en particulier par l'étude de la succession des crises financières à partir des années 1980. De même, le retour de notions comme celle de classe dominante n'est pas analysé comme le retour d'une situation de domination qui aurait auparavant mystérieusement disparu, mais comme le retour de la mobilisation de concepts pour décrire des situations qui s'ancrent, elles aussi, dans les mutations du fonctionnement de l'économie depuis plusieurs décennies.

Mon questionnement porte, dans le cadre ainsi défini, sur les analyses formulées pour rendre compte de la crise en incriminant le 
comportement des classes dominantes. Ces analyses représentent des mises à l'épreuve du système pénal, qui se distinguent pour partie des analyses inscrites dans le champ de la sociologie de la déviance. D'une part, elles reprennent l'analyse savante de la gestion différentielle des illégalismes par les institutions pénales (Lascoumes et Nagels, 2014). D'autre part, elles formulent des analyses que l'on peut qualifier de profanes, parce qu'elles passent moins par la qualification délictueuse ou pénale de comportements que par des idées plus générales: telle pratique est anormale, telle autre s'apparente à du «vol», telle autre encore se voit assimilée à de la violence. Une distinction opérée par Boltanski (2009) permet de rendre compte de la différence entre ces registres de critiques. Parmi les épreuves que doivent subir les institutions, il distingue une épreuve de vérité, mode de justification de l'institution par elle-même, d'une épreuve de réalité, par laquelle l'institution est prise au mot par ses détracteurs, par exemple pour montrer qu'elle ne fait pas ce qu'elle dit faire, ou qu'elle n'obtient pas les résultats qui sont censés justifier son existence. Une épreuve de réalité typique est la confrontation des principes de l'institution judiciaire et de son fonctionnement réel. En matière de sociologie de la délinquance des classes dominantes, la question qui traverse une telle épreuve de réalité est de comprendre comment, à partir de la formulation de principes universels - la loi pénale ne reconnaît pas les classes sociales -, se créent, sous des formes diverses, des inégalités de traitement et de sanction.

Boltanski (2009) désigne une autre forme d'épreuve comme une épreuve existentielle: «Au moins quand elle parvient à se formuler et à se rendre publique, elle dévoile l'incomplétude de la réalité et même sa contingence en puisant dans le flux de la vie des exemples à même de faire vaciller ses assises et de la mettre en cause» (p. 170). Le recours à des qualifications pénales pour décrire les activités des classes dominantes est le signe d'une telle épreuve. Il ne s'agit pas tant de chercher à faire correspondre des agissements et des délits, comme «l'abus de bien social» ou le «trafic d'influence», que de mobiliser des qualifications plus générales de justice et d'injustice. Des analyses et interprétations puisent dans le «flux de la vie» des comparaisons entre des comportements ordinairement réprimés et des comportements jugés légitimes des classes dominantes. Elles entendent ainsi montrer la contingence de cette répartition et, le cas échéant, la nécessité de la changer.

La ligne directrice suivie par l'article est la suivante. Je tente d'abord d'illustrer l'idée qu'une forme actuelle de la critique sociale porte sur 
la qualification comme crime des pratiques économiques des classes dominantes (1). Je montre dans un second temps que, formulées en dehors de l'analyse habituelle des comportements criminels, ces analyses s'adossent à un projet scientifique qui repose moins sur les outils de compréhension de la déviance que sur la manière dont les pratiques dénoncées structurent les comportements des classes dominantes (2). Une telle caractérisation pose question dès lors qu'il s'agit d'envisager les réactions politiques et sociales qu'il convient d'apporter à ces actes. Pour cette raison, dans un troisième temps, j'essaye de réfléchir aux sens qui peuvent être donnés à l'idée de sanction, dans un cadre pénal et au-delà (3).

\section{La qualification criminelle des pratiques économiques des dominants}

Des analyses sociologiques et économiques empruntent des qualifications délinquantes ou criminelles pour qualifier les pratiques économiques des classes dominantes. Il n'est pas nouveau que les normes pénales en la matière fassent l'objet de discussions. La qualification juridique d'un acte comme criminel est, comme le sont les institutions chargées des poursuites et de la répression, inscrites dans une histoire changeante et conflictuelle. La sociologie de la déviance montre que des groupes se mobilisent pour agir sur la formulation de la loi même, par exemple lorsque des entreprises font du lobbyisme pour amoindrir le caractère contraignant de projets de réglementation (Tillman, 2009). Le contexte de crise économique, en remettant au centre de l'intérêt public les règles de fonctionnement du capitalisme, opère un élargissement du spectre d'interrogation. Des chercheurs et des militants renouent avec l'idée que si des processus capitalistes ont conduit à une crise sociale profonde, c'est qu'ils doivent, en eux-mêmes, être qualifiés de manière pénale. Ces qualifications prennent trois formes principales: le vol par soustraction de revenus à la fiscalité; l'extorsion par le mécanisme de la dette; et la violence en raison des conséquences sociales de décisions économiques.

La première forme consiste à analyser la formation des richesses en déplaçant la frontière des gains licites et du vol. Un ressort classique de l'analyse de la gestion différentielle des illégalismes consiste à montrer les différences dans les réactions de l'État en matière d'infractions aux règles de la fiscalité. Spire et Weidenfeld (2015) rappellent ainsi qu'«en 
France, la fraude fiscale est l'un des délits les moins sévèrement sanctionnés par les juridictions pénales, même en comparaison d'autres crimes en col blanc. Les peines de prison ferme sont rares» (p. 16). Mais, en matière de soustraction des capitaux et revenus à la fiscalité, des auteurs élargissent l'angle d'analyse: les voleurs sont autant les fraudeurs que ceux qui aménagent des règles fiscales permettant, avec l'apparence de la légalité, de contourner la fiscalité des États. De tels raisonnements sont tenus pour caractériser les pratiques fiscales d'États qui se mettent en concurrence avec leurs voisins. C'est par exemple ce qu'explique Piketty (2013) concernant les régimes fiscaux de certains États européens, comme le Luxembourg: "On ne peut pas s'enrichir par le libre-échange et l'intégration économique avec ses voisins, puis siphonner leur base fiscale en toute impunité. Cela s'apparente à du vol pur et simple» (p. 847). Piketty reprend cette position en commentant un film (Le prix à payer, sorti en 2015) qui analyse les ressorts de cette mise en concurrence fiscale: «Ils appellent cela l'économie de marché, mais c'est du vol.» Sa critique repose sur la qualification d'un vol par la soustraction à la fiscalité, permise par une compétition entre États pour revoir les taux d'imposition à la baisse.

Une deuxième qualification visant à déplacer la frontière entre licite et illicite repose sur l'idée d'extorsion. Il s'agit de qualifier le rapport de force violent qui préside à des échanges économiques inégaux. De telles analyses se déploient principalement concernant le rapport des États et de leurs contribuables à la dette publique. Il s'agit d'un thème critique qui traverse les tentatives pour auditer et décomposer les dettes et identifier les bénéficiaires des intérêts de la dette (Collectif pour un audit citoyen de la dette publique, 2013). Une pétition pour un «audit citoyen» concernant la dette publique française demande par exemple: "Cette dette a-t-elle été tout entière contractée dans l'intérêt général, ou bien peut-elle être considérée en partie comme illégitime?» Le travail, traduit et discuté dans de nombreux pays, de l'anthropologue Graeber (2014) estime que «le langage de la dette permet de justifier une relation de pouvoir arbitraire» (paragr. 3). De manière significative, il prend en exemple d'un tel fonctionnement une organisation criminelle: "Parler de dette devient un moyen pour décrire des relations inégales. Les mafieux ont compris cela: ils utilisent souvent le terme de dette, même si ce qu'ils font est en réalité de l'extorsion» (paragr. 5). L'opération critique est double: elle fait peser sur les détenteurs de la dette une qualification violente tout en constatant qu'ils ne sont pas, au 
contraire des délinquants ordinaires et des opposants politiques, des cibles de la justice pénale.

Une troisième forme de qualification pénale des pratiques des classes dominantes porte sur l'idée de violence, dans les liens entre les décisions des élites économiques et la vie des personnes qui sont touchées par ces décisions. Elle est claire dans le titre de l'ouvrage des PinçonCharlot, La violence des riches (2013). Les auteurs dénoncent l'idée que les pratiques des classes dominantes, par leur aspect technique et financier, seraient des actes sans victimes. Pour eux, il s'agit, à l'inverse, de qualifier une violence à distance: par opposition à la violence interpersonnelle qui conduit devant les tribunaux, la violence des riches s'exerce par leur pouvoir sur les entreprises et les salariés. PinçonCharlot (2013) explique sa logique de mise en accusation «avec l'histoire d'une entreprise de métallurgie, qui était le numéro un mondial des pôles d'alternateur pour automobiles»:

Une petite entreprise familiale avec 400 salariés, à qui les banques ont arrêté de prêter de l'argent, du jour au lendemain, et demandé des remboursements, parce que cette PME refusait de s'ouvrir à des fonds d'investissement. L'entreprise a été placée en redressement judiciaire. Un fonds de pension l'a récupérée pour un euro symbolique, et, en deux ans, a pillé tous les savoir-faire, tous les actifs immobiliers, puis fermé le site. 400 ouvriers se sont retrouvés au chômage. C'est un exemple parmi tant d'autres! Si vous vous promenez dans les Ardennes aujourd'hui, c'est un décor de mort. (p. 2)

Pour Pinçon-Charlot (2013), une décision de fermeture d'usine, «c'est une violence inouie [qui] brise des vies, qui atteint les gens au plus profond de leur corps, de leur estime, de leur fierté du travail» (p. 2). Elle s'inscrit ainsi dans la dénonciation non seulement de la violence comme brutalité, mais dans la caractérisation des conséquences à long terme de ces actions sous les traits du traumatisme, habituellement utilisés pour décrire les conséquences de crimes comme les violences sexuelles subies dans l'enfance (Fassin et Rechtman, 2007).

La critique de cette violence à distance s'incarne dans des slogans tels que «l'austérité tue», qui veulent montrer les effets des politiques et décisions économiques en mettant l'accent sur leurs effets délétères sur la vie des personnes touchées. Il s'agit parfois directement de suicides causés par l'entrée dans la pauvreté. Par exemple, le 4 avril 2012, Dimitris Christoulas s'est rendu place Syntagma, en plein centre-ville d'Athènes. Il a gravi les marches du parlement, placé une arme contre 
sa tempe et déclaré: «Je ne me suicide pas. Ce sont eux qui me tuent.» Des études qui montrent des effets de la pauvreté sur la santé et l'espérance de vie des classes populaires donnent à cette idée une portée plus générale. C'est l'argument par exemple de l'ouvrage de Stuckler et Basu (2014). Ils reprennent la logique d'une méthode économique de détermination des effets de politiques publiques: les quasi-expérimentations, c'est-à-dire les situations dans lesquelles un état de départ relativement comparable dans deux cas est transformé de manière différente par une action publique. En comparant les évolutions depuis le début de la crise financière de pays ayant suivi des politiques radicalement opposées, les auteurs entendent les effets mortifères des politiques économiques d'austérité. Pour ce faire, ils comparent l'Islande, qui a décidé de ne pas payer la dette de ses banques et de relancer son économie en renforçant ses politiques sociales, et la Grèce, soumise à des politiques d'austérité répétées. Ils illustrent les conséquences sanitaires mortelles de cette orientation politique, en montrant par exemple que, «à la suite des coupes drastiques opérées dans les budgets alloués au sud du pays, une épidémie de virus du Nil occidental a éclaté en août 2010, tuant soixante-deux personnes» (Stuckler et Basu, 2014, p. 3).

Ces critiques des pratiques des classes dominantes comme vol, extorsion et violence empruntent des arguments différents mais fonctionnent selon une logique semblable: la description de dommages (sommes en jeu, torts causés, nombre de morts) et la comparaison de la manière dont la société traite ces dommages au traitement judiciaire ordinaire de la délinquance. Ces formes de critiques sociales ne sont pas nouvelles. Au $\mathrm{xIX}^{\mathrm{e}}$ siècle, un mot d'ordre célèbre du socialiste Proudhon estime que «la propriété, c'est le vol». Après Mai 68, les mouvements d'extrême gauche qualifient leurs adversaires politiques selon des catégories pénales. Par exemple, les militants maoïstes politisent un accident mortel dans une mine en organisant le procès symbolique de ses responsables. Pour cela, ils cherchent à montrer que leurs agissements sont criminels, parce qu'ils provoquent sciemment des accidents mortels (Bérard, 2013b).

Le retour de ces analyses sur le devant de la scène est symptomatique de ce qu'on pourrait appeler des moments critiques, au double sens de crise $\mathrm{du}$ fonctionnement du capitalisme et de développement de formes renouvelées d'analyses critiques de ce fonctionnement. Mais ces expressions peuvent avoir des sens différents. Elles pourraient ainsi former un simple révélateur rhétorique du degré de radicalité politique de celui 
qui les formule: ce qui est dénoncé comme crime est ce qui jugé inacceptable. Les auteurs et mouvements modérés désigneraient ainsi ce qu'ils jugent comme excès par distinction avec ce qu'ils jugent légitime, tandis que ceux qui pensent qu'il faut renverser le capitalisme le placeraient tout entier du côté du vol et de la violence. Il me semble que les auteurs que j'ai cités vont au-delà de cette dénonciation: ils estiment que le lien entre les classes dominantes et la pratique d'illégalismes est un trait structurel qui permet de spécifier l'exercice contemporain du pouvoir. C'est ce que je vais essayer de montrer dans mon deuxième point.

\section{La domination des règles}

La crise économique a vu se multiplier les figures de voyous financiers. De Jérôme Kerviel à Bernard Madoff, ces personnalités ont incarné les dérives du système. Certaines ont été traduites devant les tribunaux et condamnées à de lourdes peines. Pourtant, les positions critiques que j'ai mobilisées n'accordent pas à ces condamnations une place analytique importante. C'est par exemple le sens de l'exercice de pensée que propose Lordon (2014a), qui récuse explicitement une orientation qu'il qualifie de «criminologique» :

Prendre la mesure de l'inanité analytique du point de vue criminologiquemédiatique requiert, par exemple, de se livrer à une simple expérience de pensée contrefactuelle demandant si la crise financière aurait été évitée si les fâcheux [Jérôme Kerviel et Bernard Madoff] n'avaient pas été là. Sauf passion du bouc émissaire et paranoïa en roue libre, la réponse est évidemment non, et les individus délinquants par conséquent renvoyés à leur juste statut: même pas épiphénoménal, simplement secondaire. (paragr. 2)

Pour déplacer l'attention au-delà de ces cas médiatiques, Lordon invite à la compréhension des liens structurels entre illégalismes et pratiques de classes dominantes contemporaines. Ce lien est formulé par Boltanski (2009) lorsqu'il réfléchit aux enjeux de la reprise d'une sociologie des classes sociales.

L'analyse de Boltanski est significative à plusieurs égards. D'une part, elle marque ce qui est souvent décrit comme le retour de cet auteur à la «sociologie critique» (Lordon, 2013), qui se lit dans le livre qui accompagne la réédition de l'article coécrit avec Bourdieu en 1976, «La production de l'idéologie dominante» (Boltanski, 2008), puis dans De la critique (2009). Son intérêt repose sur une tentative de caractérisation du présent par l'analyse du rapport à la loi des classes dominantes. 
Lorsqu'il caractérise la «culture» nouvelle des responsables, Boltanski (2008) juge que le «point central» concerne justement leur «relation à la règle». Dans le capitalisme d'après la Seconde Guerre mondiale, estime-t-il, «même si les Lois étaient forgées, pour l'essentiel, dans le creuset de la classe dominante, il n'en restait pas moins que chaque acteur, pris individuellement, pouvait se faire croire à lui-même que son action était contrainte par des règles impersonnelles, et, en quelque sorte, transcendantes». Or, selon Bolstanki (2008),

l'apprentissage et l'exercice du management, surtout dans ses modalités nouvelles qui fait une large place à une grande diversité d'instances non étatiques de production de normes, ont ouvert les yeux des «responsables» en ne leur permettant plus d'ignorer qu'ils étaient les fabricants des règles qu'ils imposaient aux autres. (p. 169)

Ce point central est de l'ordre du «savoir d'initié». S'il était public, se poserait

la question de savoir (...) pourquoi certains, le plus grand nombre, doivent considérer les règles comme quasiment sacrées, les respecter, sous peine de sanctions, (...) tandis que d'autres peuvent au contraire les ajuster au gré d'intérêts qu'il est toujours loisible de présenter, lorsque des «responsables» ayant été un peu loin se trouvent pris sur le fait, comme orientés malgré tout vers le bien commun. (Boltanski, 2008, p. 171)

Une telle analyse donne en somme comme tâche centrale à la critique sociale le dévoilement public du secret bien caché des relations entre domination économique et sociale et jeu avec les règles. Cette idée de Boltanski (2008) est souvent citée pour montrer la fécondité d'un retour à une vision sociologique de la délinquance des classes dominantes (Spire, 2013). Même si elles divergent, les analyses qui dénoncent les pratiques des classes dominantes «comme des crimes» les considèrent en même temps comme des traits non pas déviants mais ordinaires et liés à des structures de pouvoir. Zucman (2013) montre que la fraude fiscale liée aux capitaux placés dans des paradis fiscaux n'est pas marginale mais concerne 7 à $8 \%$ de la richesse mondiale. Ceux que Piketty désigne comme des voleurs ne sont rien de moins que les États euxmêmes lorsque ceux-ci se lancent des politiques de concurrences fiscales et lorsqu'ils ménagent, au sein de leur propre système, des espaces de négociation discrétionnaire des règles. La quasi-simultanéité de la nomination de Jean-Claude Juncker à la présidence de la Commission européenne et de la révélation des pratiques fiscales occultes du Luxembourg illustre bien ce type de situation (Piketty, 2014). 
Les États «voleurs» ne sont pas les seuls bénéficiaires de ces pratiques: en attirant les entreprises par une fiscalité avantageuse, ils leur font d'abord faire des profits accrus. La critique vise alors moins des déviances que les pratiques fiscales des plus grandes entreprises, soutenues par les plus grandes banques. Selon Lordon (2014a),

la généralisation des comportements crapuleux finirait presque par faire croire à l'existence non pas de simples déviations récurrentes, idée en soi tendanciellement oxymorique, mais à un véritable business model, où une partie du dégagement de profit est très délibérément remise à l'exploitation de situations frauduleuses. (paragr. 4)

Le jeu avec les règles s'accompagne de stratégies sophistiquées pour rester, ou avoir l'apparence de rester, du bon côté du droit. En effet, «si la mise à distance - voire la délégitimation - de la règle constitue une caractéristique des classes dominantes, cette tendance s'accompagne d'un souci de sécuriser leurs pratiques lorsqu'elles sont à la lisière du droit: chaque fois qu'il existe un risque de se voir reprocher d'enfreindre la loi, les membres des classes dominantes prennent soin de se mettre préalablement à l'abri de toute sanction» (Spire, 2013b, p. 10).

Ces critiques essayent de caractériser des actes qui sont propres au «champ du pouvoir» (Denord et al., 2011). Cette notion vise à dépasser une conception «pluraliste» selon laquelle «pouvoirs et contre-pouvoirs organiseraient la confrontation équilibrée d'intérêts divergents», et dans le cadre de laquelle «patrons, professionnels libéraux, serviteurs de l'État, responsables politiques, journalistes, universitaires, intellectuels ou artistes font l'objet d'analyses distinctes malgré une large communauté d'origines, de formations ou de lieux de sociabilité». Pour ces auteurs, les affaires judiciaires donnent précisément à voir un champ du pouvoir que des approches trop segmentées ne peuvent pas saisir. Par exemple, «l'affaire Woerth-Bettencourt ${ }^{2}$ - un scandale politico-financier survenu en 2010 - se révèle si caricaturale qu'elle en devient exemplaire. Elle tisse un inextricable écheveau de relations entre des univers sociaux que l'idéal pluraliste souhaiterait voir autonomes si ce n'est strictement séparés» (Denord et al., 2011, p. 26). L'affaire rend visibles les échanges entre des responsables politiques, des gestionnaires de fortunes privées, des juristes chargés du montage des opérations financières et des artistes

2. Il s'agit d'une affaire financière complexe portant sur les sommes données, alors qu'elle pouvait se trouver en état de faiblesse, par Liliane Bettencourt, actionnaire milliardaire de l'Oréal. 
qui bénéficient de libéralités. En analysant les formations, trajectoires et relations des personnes inscrites au Who's who, l'article caractérise «la fraction dominante du champ du pouvoir» comme «une zone-carrefour, située à l'intersection entre la politique, l'administration, le capitalisme d'État, le secteur privé et le capitalisme familial». C'est à cette intersection qu'on peut situer les protagonistes de l'affaire Bettencourt. Ceux-ci ont les traits que donne Wagner (2011) des «classes dominantes à l'épreuve de la mondialisation». Elle décrit également comme un trait spécifique leur capacité «à intervenir sur le processus de production de la loi fiscale, indissociable de l'intrication entre le champ des élites économiques et celui du pouvoir politique» (p. 8).

Stuckler et Basu cherchent à clarifier le statut des responsables qui sont désignés par des formules comme «l'austérité tue». Ils estiment que «les politiques économiques ne sont ni des agents pathogènes ni des virus qui provoquent directement la maladie, mais la «cause des causes » : le facteur sous-jacent qui détermine qui sera exposé aux plus grands risques sanitaires» (2014, p. 3). La notion de «cause des causes» est intéressante parce qu'elle marque une distinction avec le fonctionnement ordinaire du système pénal. Elle détache le questionnement de l'intentionnalité, de la responsabilité morale et pénale pour se placer en ce qui concerne les décisions structurelles dont les conséquences sont rendues visibles par les analyses des chercheurs. Pour cette raison, l'incrimination des «causes des causes» pose une question pénale délicate: comment penser les relations entre ces analyses sociales générales et l'exercice de sanctions pénales? C'est le point que j'aborde dans une troisième partie.

\section{Les usages de la sanction}

Un trait commun, on l'a vu, des analyses des comportements des classes dominantes comme des crimes, est de comparer leur impunité à la sévérité que la justice pénale réserve à d'autres comportements. Mais cela signifie-t-il qu'il faille faire subir aux classes dominantes le sort habituellement réservé aux auteurs d'infractions issus des classes populaires? On peut donner une formulation plus savante de cette question à l'aide de deux notions de théorie de la peine et de sociologie de la déviance. La rationalité pénale moderne est définie par Alvaro Pires comme le cadre théorique dans lequel se déploie la pénalité et qui lui donne ses traits fondamentaux (hostile, négative, abstraite, atomisante) 
(Debuyst, Digneffe et Pires, 1998). Les filières pénales, analysées par la sociologie de la déviance (Aubusson de Cavarlay, 1985; Lascoumes, 1986; Robert, 1977), permettent de comprendre l'ensemble des mécanismes d'orientation et de sélection qui jalonnent la chaîne pénale et expliquent le traitement différencié des illégalismes selon les types de délit et l'origine sociale de leurs auteurs. Ces notions descriptives peuvent permettre de formuler une question normative: faut-il renverser les filtres de sélection des filières pénales et orienter vers d'autres cibles sociales les rigueurs de la rationalité pénale? Ou donner à l'idée de sanction une signification qui excède la dimension pénale?

L'analyse structurelle des relations entre illégalismes et classes dominantes peut donner à leur qualification criminelle une dimension principalement symbolique: il s'agit alors d'une modalité d'interpellation et de mobilisation. Cette dimension symbolique plus que directement pénale est celle, par exemple, de la dénonciation des «rémunérations obscènes» analysées par Steiner. Celui-ci explique le choix du terme par la «violence verbale» qu'il contient: obscène signifie ici «qui blesse ouvertement, qui révolte» ou encore «qui choque par sa démesure». De même, dire qu'une politique fiscale est du «vol» ou qu'une politique d'austérité «tue» est une représentation «qui blesse ouvertement».

Pour autant, les auteurs n'en restent pas à cette dimension symbolique et, en bonne logique pénale, se demandent s'il faut lier la répression à la dénonciation. Les réponses apportées à cette question sont contrastées. La critique du recours à l'incarcération comme outil de résolution des problèmes sociaux fait partie des positions politiques des auteurs et mouvements de gauche. La figure de Foucault est importante à ce titre: si ses analyses sur la gestion différentielle des illégalismes sont mobilisées (Amicelle, 2014), il demeure plus connu pour le lien qu'il a établi entre un travail généalogique sur l'histoire de la prison et l'implication dans des mouvements politiques de critique de l'incarcération. Dans les années 1970, les tentatives maoïstes de renversement de la sévérité pénale contre la bourgeoisie ont vite été disqualifiées par l'émergence de mouvements militants contre la répression (Bérard, 2013a). Plus près de nous, dans les années 1990, l'incarcération de quelques responsables politiques a été une occasion de politisation des conditions de détention, par des figures comme Pierre Botton, aujourd'hui encore actif dans la recherche de nouvelles formes de sanction. L'incarcération de membres des élites est plus souvent l'occa- 
sion d'une reprise de la dénonciation de la condition carcérale que de la célébration d'un acte de justice. Dans l'espace de la critique sociale, du constat de l'impunité des classes dominantes à la demande de leur sanction par la prison, la conséquence n'a rien d'évident.

Des chercheurs défendent l'idée d'une transformation radicale de l'action pénale, retournant ses armes les plus sévères - de longues peines de prison - contre les délinquants en col blanc (présentés, par exemple dans Lascoumes et Nagels, 2014). Des exemples de condamnation de ce type viennent souvent des États-Unis. Mais elles prennent place dans le contexte d'un système pénitentiaire gigantesque, dans lequel la sévérité extrême des peines continue de s'abattre prioritairement sur les plus pauvres. À l'inverse, la description de l'action disciplinaire d'organes intermédiaires entre les auteurs d'infraction et la justice pénale met en lumière des enjeux qu'il n'est pas possible d'évacuer d'un trait de plume: faut-il préférer la brutalité de la peine au pouvoir de contraindre des acteurs à rentrer dans l'ordre par d'autres moyens? En ce qui concerne la délinquance des classes populaires, la volonté d'un usage moindre de la prison s'appuie souvent sur l'idée de revaloriser des formes d'action en amont du recours au système pénal ou évitant le recours aux sanctions les plus lourdes.

Cela ne signifie pas que la question de l'action pénale soit considérée comme marginale. Elle peut prendre différents sens. Le premier repose moins sur la menace de la prison que sur celle de la stigmatisation. Par exemple, une tribune publiée dans Le Monde, par Spire et Weidenfeld (2015) a pour titre: «Contre la fraude fiscale, renforçons la sanction pénale». Cela étant, le titre dissimule en partie la position des auteurs. L'article mentionne «la réticence des juges à envoyer des personnes bien insérées dans des prisons déjà surpeuplées». Selon eux, il ne s'agit pas tant de punir de nombreux fraudeurs par la prison que de les menacer de sanctions et de la publicité de ces sanctions (naming and shaming). Ils signalent, que «menée sans précautions, cette politique de stigmatisation est néanmoins discutable et discutée», parce qu'une «fraude répandue et étalée au grand jour pourrait la banaliser, en même temps que renforcer le sentiment d'injustice» (Spire et Weidenfeld, 2015, p. 16). Ils retiennent cependant l'idée d'une «mise en récit pénal» qui rende visibles les mécanismes des illégalismes et s'oriente «vers la recherche des organisateurs et les facilitateurs de la fraude». La dimension symbolique du recours à la pénalité est en ce sens un outil de prévention générale. 
Un autre usage envisagé de la sanction pénale est d'en faire une arme dans une négociation entre les États et les fraudeurs. Lors des scandales qui sont révélés par la publication de listes de comptes en Suisse dissimulés aux administrations fiscales nationales, il est souvent fait mention du fait que la situation a été «régularisée», c'est-à-dire que l'argent a été rapatrié moyennant une pénalité financière. Zucman (2013) propose une forme plus radicale de négociation. Étant donné que la dissimulation constitue une infraction pénale pouvant déboucher sur des poursuites et des sanctions, les États pourraient échanger l'absence de poursuite contre la saisie de la totalité de l'argent dissimulé (Zucman, 2013). La menace pénale joue ainsi le rôle d'outil dans l'établissement d'un rapport de force. Mais le livre de Zucman (2013) montre bien que ce rapport de force est plus fondamentalement dépendant de la capacité des États à établir de manière certaine la cartographie du capital détenu par leurs citoyens. Cette transparence, qui nécessiterait des réformes importantes, est le préalable à la négociation, sans quoi les États ne peuvent que demander le retour des fonds qu'ils ont été capables de repérer et qui ne forment qu'une faible part de la «richesse cachée des nations».

Un troisième usage élargit la question de la sanction à celle de la puissance publique. Lordon (2014a) donne par exemple à l'idée de sanction le sens du rappel de la puissance de l'État. C'est ainsi qu'il analyse par exemple l'amende infligée par la justice américaine à la plus grande banque française, BNP Paribas, pour des transactions irrégulières dans des pays sous embargo américain:

S'il y a bien une leçon à tirer de l'affaire BNP Paribas, ça n'est pas tant que les banquiers néolibéraux sont des fripouilles, aussi bien au sens du Code pénal que de la nuisance sociale, c'est que la puissance publique, pourvu qu'elle le veuille, n'a ni à passer sous le tapis ni à céder à tous les ultimatums du capital. (paragr. 19)

Contre la défense de la banque par les responsables politiques français au nom du patriotisme économique, l'économiste Giraud (2014) juge la sanction «légitime». Il estime que de telles actions devraient s'étendre: «s'agissant, par exemple, des nombreux paradis fiscaux où cette banque est implantée», car son "impunité est un très mauvais signal adressé aux passagers clandestins en col blanc qui, sur notre continent, esquivent leurs responsabilités de citoyens-contribuables» (paragr. 5). Au nom de la même idée, il estime que «les autorités européennes, grecques, italiennes... pourraient sanctionner Goldman Sachs 
pour avoir truqué les comptes publics grecs qui ont permis à Athènes d'entrer dans la zone euro, et JP Morgan pour avoir vendu des prêts toxiques en Italie» (Giraud, 2014, paragr. 7).

Ces réactions s'opposent aux positions exprimées par le gouvernement français. Lorsque la sanction a été infligée, celui-ci a fait valoir qu'elle était disproportionnée. Il a porté cet enjeu sur le plan de discussions diplomatiques pour dénoncer une «décision injuste et unilatérale», selon les termes du ministre français des Affaires étrangères. Giraud (2014) et Lordon (2014a) reprennent cette idée pour en tirer la conclusion inverse: des sanctions mettant en jeu de tels montants sont évidemment des décisions souveraines qui manifestent, contre l'idée du retrait de l'État de la régulation de la sphère financière, qu'il est possible qu'il s'y intéresse et qu'il impose des mesures qui ne soient pas anecdotiques pour les entreprises concernées. Le sens de la sanction comme réaffirmation de la puissance publique élargit de fait son rayon d'action bien au-delà du champ de la justice pénale. Elle fait entrer cette idée comme modalité des rapports de force qui peuvent s'incarner dans des politiques fiscales ou douanières. Par exemple, lorsqu'il réfléchit aux moyens d'avancer en matière d'échanges d'informations entre les banques et les États, Piketty (2013) mobilise l'idée d'une sanction: «il est probable que la seule façon d'obtenir des résultats tangibles est d'imposer des sanctions automatiques non seulement aux banques mais également aux pays qui refuseraient d'étendre dans leur droit interne les obligations de transmission automatique à tous les établissements basés sur leur territoire»(p. 850). De même, lorsqu'il réfléchit aux manières de transformer radicalement le fonctionnement $\mathrm{du}$ monde financier, Lordon (2014a) avance des idées de sanction : «les règles (...) de la nouvelle régulation posées, toute infraction sérieuse sera aussitôt sanctionnée par une nationalisation-saisie, soit une expropriation sans indemnité aucune des actionnaires» (paragr. 22).

Les options politiques défendues par Piketty (2013) et Lordon (2014a) sont très différentes, de l'impôt mondial sur le capital à la saisie des banques. Un lien logique lie cependant leur usage de l'idée de sanction: les pratiques dénoncées sont définies comme étant des pratiques normales, au sens d'ordinaire, et considérées comme légitimes par leurs protagonistes. S'il faut trouver des actions à même de redresser ces conduites, elles empruntent à l'idée de sanctions, mais celles-ci doivent se placer à un niveau de puissance qui rende crédible leur influence sur des acteurs eux-mêmes puissants, politiquement et écono- 
miquement. C'est à ce moment que des divergences apparaissent et, pourrait-on dire, que les questions politiques sérieuses commencent. Quel degré de transformation de l'économie capitaliste faut-il viser? Les institutions actuelles, en particulier européennes, peuvent-elles être le cadre de ces régulations, sanctions et réappropriations? Ou, sont-elles totalement capturées par les intérêts des classes dominantes (Chavagneux et Philiponnat, 2014) et structurellement conçues pour être réfractaires à ces transformations (Lordon, 2014b)?

Il n'est évidemment pas question d'essayer de répondre ici à ces questions. Mais elles signalent à mon sens un point qui peut intéresser la criminologie critique: ce n'est pas parce que l'économie et la sociologie des classes dominantes analysent des pratiques illégales et proposent des mécanismes de sanctions qu'elles en appellent au premier chef à l'usage du système pénal. Dans leur optique, les relations structurelles des élites à la loi sont trop importantes pour être laissées à la justice pénale, pensée et mise en œuvre comme un système de traitement des déviances. Penser ces questions dans un cadre élargi est peut-être un petit pas en avant pour dépasser les contradictions qui naissent lorsqu'il s'agit de faire tenir ensemble la critique de l'impunité des élites et la critique des instruments de la répression policière, judiciaire et carcérale.

\section{Conclusion}

Lorsqu'elles décrivent les maux du capitalisme contemporain, la sociologie critique et l'économie hétérodoxe empruntent régulièrement au vocabulaire du crime des descriptions de pratiques qu'elles jugent condamnables. La qualification pénale des actions des classes dominantes s'inscrit dans la volonté générale de rompre avec ce que Tévanian et Tissot (2010) nomment l'euphémisation et l'occultation de la «violence des dominants». Il ne fait pas de doute que la mise en place des options politiques défendues par les auteurs sur lesquels je me suis appuyé donnerait lieu à des dénonciations reprenant, en sens inverse, les mêmes formes de critique: de tels impôts, droits de douane ou expropriations seraient dénoncés à l'autre bout du spectre politique comme du vol, de l'extorsion et de la violence. En ce sens, la mobilisation du vocabulaire de la déviance est-elle plus qu'un trait rhétorique, parmi d'autres, pour qualifier ou disqualifier des revendications? D'une certaine façon, toute expression militante s'inscrit dans la défense de ce qu'elle considère comme juste d'une manière plus générale, non pas 
seulement sur le plan des crimes et délits. Il est logique que la dénonciation de situations d'injustice emprunte au vocabulaire de la déviance et de la criminalité, et que, situées dans différents espaces sociaux, les solutions envisagées empruntent à des registres d'action qui excèdent ceux de la justice pénale.

La dénonciation de crimes dans la littérature critique est pourtant davantage qu'un enjeu rhétorique. Elle a l'ambition d'une analyse structurelle d'un trait du champ du pouvoir, ainsi décrite par exemple par Wagner (2011): «le rapport relativiste des dominants à la règle, notamment fiscale, est exemplaire de cette relation à la fois instrumentale et distanciée à l'État, et pourrait bien être une des dimensions de ce nouvel état du rapport de force au sein du champ du pouvoir» (p. 8). On pourrait dire ainsi que plus les classes dominantes sont considérées comme criminelles, moins elles sont considérées comme déviantes. Le conditionnel employé par Wagner signale le caractère d'hypothèse d'une telle idée, qui demeure à approfondir et préciser. Mais il me semble qu'elle illustre l'intérêt de réfléchir à la criminalité des élites à partir des études sur les élites en général, et d'analyser en conséquence les enjeux politiques liés à ces pratiques comme des questions politiques portant sur les formes contemporaines de la domination politique et économique. La manière dont ces auteurs manipulent la notion de sanction illustre l'élargissement nécessaire à la sortie des apories liées à l'usage de la justice pénale ordinaire comme mode de transformation de situations qui excèdent largement son pouvoir.

Il y a trente ans, Brodeur (1984) dressait un panorama des critiques apportées à la criminologie marxiste. Au-delà des diverses options théoriques des auteurs étudiés ressortait nettement que le vocabulaire des classes sociales, de la détermination en dernière instance, etc. masquait une incapacité à rendre compte des données empiriques de la recherche selon ces catégories trop larges ou employées de façon trop systématique. Brodeur écrivait à un moment où cette criminologie radicale déclinait, symptôme, parmi d'autres, de l'entrée dans les années 1980 et d'une autre conjoncture intellectuelle. Aujourd'hui, Tombs et Whyte (2015) datent aussi du début des années 1980 le reflux des analyses liant la criminalité au fonctionnement plus général du capitalisme. De même, en France, Lordon (2013), après d'autres, caractérise le «changement tectonique qui a affecté la vie intellectuelle à la fin des années 1970 et sous le régime duquel nous vivons toujours» par la raréfaction, si ce n'est la disparition, des analyses mobilisant les notions 
de structure et de domination» (p. 124-125). Le contexte actuel voit la reprise d'un intérêt pour des analyses qui mobilisent, sous des formes renouvelées, les catégories de classes sociales et de domination. Une grande question des années 1970 a été d'articuler les formes générales de l'exploitation industrielle - les usines - et les institutions disciplinaires chargées des «mauvais pauvres»- les prisons. Peut-être la crise financière et l'attention accrue portée par différentes branches des sciences sociales aux pratiques des élites sont-elles l'occasion de retrouver, par le haut de la hiérarchie sociale cette fois, le lien entre les sociologies des classes sociales et des illégalismes.

\section{Références}

Amicelle, A. (2015). «Deux attitudes face au monde»: La criminologie à l'épreuve des illégalismes financiers. Cultures \& Conflits, 2 (94-95-96), 65-98.

Aubusson de Cavarlay, B. (1985). Hommes, peines et infractions, la légalité de l'inégalité. L'Année sociologique, 35(3), 275-309.

Bérard, J. (2013a). La justice en procès. Les mouvements de contestation et le système pénal (1968-1983). Paris, France: Presses de Sciences-Po.

Bérard, J. (2013b). Tordre ou briser le bâton de la justice? Les mouvements de l'après-68 et les illégalismes des dominants, entre justice populaire et refus de la pénalité (1968-1972). Champ pénal/Penal field, X. Repéré à URL: http://champpenal.revues.org/8582.

Boltanski, L. (2008). Rendre la réalité inacceptable, à propos de "La production de l'idéologie dominante». Paris, France: Editions Demopolis.

Boltanski, L. (2009). De la critique, précis de sociologie de l'émancipation. Paris, France: Gallimard.

Boltanski, L. (2011). Une étude en noir. Tracés, 20, 49-73.

Chavagneux, C. et Philipponnat, T. (2014). La capture. Où l'on verra comment les intérêts financiers ont pris le pas sur l'intérêt général et comment mettre fin à cette situation. Paris, France: La Découverte.

Collectif pour un audit citoyen de la dette publique. (2013). Mobilisation pour un audit citoyen de la dette publique. Repéré à http://www.audit-citoyen.org/? page_id $=35$.

Debuyst, C., Digneffe, F. et Pires, A. P. (1998). Histoire des savoirs sur le crime et la peine, tome 2: La rationalité pénale et la naissance de la criminologie. Bruxelles, Belgique: De Boeck Université.

Denord, F., Lagneau-Ymonet, P. et Thyne, S. (2011). Le champ du pouvoir en France. Actes de la recherche en sciences sociales, 5(190), 24-57.

Fassin, D. et Rechtman, R. (2007). L'empire du traumatisme. Enquête sur la condition de victime. Paris, France: Flammarion. 
Giraud, G. (2012). L'Illusion financière. Paris, France: Éditions de l'Atelier.

Giraud, G. (2014, 6 juin). Dix milliards de dollars sont une petite somme pour ce monstre bancaire. L'express.fr. Repéré à http://www.lexpress.fr/actualite/ au-pire-les-actionnaires-de-bnp-paribas-ne-percevront-pas-dedividendes_1549368.html

Graeber, D. (2014, 16 février). La façon la plus simple de désobéir à la finance, c'est de refuser de payer les dettes. Bastamag. Repéré à http://www.bastamag.net/David-Graeber-La-forme-la-plus.

Lascoumes, P. (1986). Les affaires ou l'art de l'ombre. Paris, France: Le Centurion.

Lascoumes, P. et Nagels, C. (2014). Sociologie des élites délinquantes, de la criminalité en col blanc à la corruption politique. Paris, France: Armand Colin.

Lordon, F. (2008). Jusqu'à quand? Pour en finir avec les crises financières. Paris, France: Raisons d'agir.

Lordon, F. (2013). La société des affects, pour un structuralisme des passions. Paris, France: Seuil.

Lordon, F. (2014a, 8 juillet). BNP-Paribas, une affaire de géométries variables. Repéré à http://blog.mondediplo.net/2014-07-08-BNP-Paribas-uneaffaire-de-geometries-variables.

Lordon, F. (2014b). La malfaçon. Monnaie européenne et souveraineté démocratique. Paris, France: Les liens qui libèrent.

Piketty, T. (2013). Le Capital au XXI eiècle. Paris, France: Seuil.

Piketty, T. (2014, 29 décembre). Quels chocs pour faire bouger l'Europe en 2015 ?. Libération.

Piketty, T. (2015, 2 février), Ils appellent ça de l'économie de marché, mais c'est du vol. Nouvelobs.com. Repéré à http://tempsreel.nouvelobs.com/economie/ 20150127.OBS0971/piketty-ils-appellent-cela-1-economie-de-marchemais-c-est-du-vol.html.

Pinçon-Charlot, M. (2013, 5 novembre). La violence des riches atteint les gens au plus profond de leur esprit et de leur corps. Bastamag. Repéré à http:// www.bastamag.net/Monique-Pincon-Charlot-La-violence.

Pinçon-Charlot, M. et Pinçon, M. (2013). La violence des riches. Chronique d'une immense casse sociale. Paris, France: La Découverte.

Robert, P. (1977). Les statistiques criminelles et la recherche. Déviance et société. 1(1), 3-28.

Spire, A. (2013a). Comment contourner l'impôt sans s'exiler. Le Monde diplomatique.

Spire, A. (2013b). Pour une approche sociologique de la délinquance en col blanc. Champ pénal/Penal field, X, URL: http://champpenal.revues.org/8582.

Spire, A. et Weidenfeld, K. (2015, 12 février). Contre la fraude fiscale, renforçons la sanction pénale. Le Monde.

Steiner, P. (2011). Les rémunérations obscènes. Le scandale des hauts revenus en France. Paris, France: Zones.

Stuckler, B. et Basu, S. (2014). Quand l'austérité tue. Épidémies, dépressions, suicides: l'économie inbumaine. Paris, France: Autrement. 
Tévanian, P. et Tissot, S. (2010). Les mots sont importants. Paris, France: Libertalia.

Tillman, R. (2015). Making the rules and breaking the rules: the political origins of corporate corruptions in the new economy. Crime, Law and Social Change, 51, 73-86.

Tombs, S. (2015). Crisis, what crisis? Regulation and the academic orthodoxy. Howard Journal of Criminal Justice, 54(1), 57-72.

Tombs, S. et Whyte, D. (2015). Introduction to the Special Issue on 'Crimes of the Powerful'. Howard Journal of Criminal Justice, 54(1), 1-7.

Wagner, A.-C. (2011). Les classes dominantes à l'épreuve de la mondialisation. Actes de la recherche en sciences sociales, 5(190), 4-9.

Zucman, G. (2013). La richesse cachée de nations, enquête sur les paradis fiscaux. Paris, France: Seuil.

\section{Ruling classes, criminal classes? White-collar crime as figure of social critique}

ABSTRACT - This article suggests that criminologists studying white-collar crime should take into account critical evaluations of the behavior of the ruling classes, many of which have been provided by French economists and sociologists. I focus on analyses of current crises that are based on criminalizing the behavior of the ruling classes, looking at how the vocabulary of deviance has been used to describe economic and political behavior as theft, extortion, and violence and showing that these accusations involving identifying such behaviors as a major element in the practices of the dominant class. For example, the revival of critical sociology is anchored in part on an analysis of the relationship of the ruling classes with laws and standards, showing that one of their distinguishing features is to consider these rules to be fundamental for others but not for themselves. Such analyses have led to policy proposals that make use of the idea of criminal punishment. But the article shows that such interpretations also influence broader policy issues, especially in the game of power relations between states and between states and capital holders outside their control.

KEYWORDS - White-collar crime, penal justice, social critique, social classes, capitalism.

\section{Clases dominantes, clases delincuentes? El crimen económico como una figura de la crítica social}

RESUMEN - El artículo aborda la cuestión de la delincuencia de elites desplazando la atención de los trabajos criminológicos hacia aquellos de economistas y sociólogos críticos principalmente franceses dedicados a los comportamientos de las clases dominantes. La problemática se centra en los análisis formulados para dar cuenta de la crisis actual, incriminando el comportamiento de las clases dominantes. El artículo examina la movilización del vocabulario de la desviación para calificar comportamientos económicos y políticos de robo, de extorsión y de violencia. Se demuestra que estas acusaciones se basan en el análisis de esos comportamientos como una característica importante de las prácticas de las clases dominantes contemporáneas. En particular, 
el aporte de la sociología crítica se basa en el análisis de la relación singular con las leyes y normas de las clases dominantes, demostrando que su rasgo distintivo es considerar esas normas como fundamentales para otros, pero eludibles para ellas. Tales análisis llevan a propuestas políticas que hacen uso de la idea de sanción penal. Sin embargo, el artículo muestra que estos usos se manifiestan en cuestiones políticas más amplias $y$, en particular, en el juego de las relaciones de poder entre los Estados, y entre los Estados y los poseedores de capitales, que escapan de su control.

palabras clave - Criminalidad económica, justicia penal, crítica social, clases sociales, capitalismo. 